\title{
Global transcriptomic analysis reveals Lnc- ADAMTS9 exerting an essential role in myogenesis through modulating the ERK signaling pathway
}

Liqi Wang, Ting He, Xin Zhang, Yubo Wang, Kai Qiu, Ning Jiao, Linjuan He and Jingdong Yin * (D)

\begin{abstract}
Background: Long non-coding RNAs (IncRNAs) are emerging key regulators involved in a variety of biological processes such as cell differentiation and development. The balance between myogenesis and adipogenesis is crucial for skeletal muscle homeostasis in humans and meat quality in farm animals. The present study aimed to reveal the global transcriptomic profiles of adipogenic (Adi-) and myogenic (Myo-) precursors derived from porcine skeletal muscle and identify IncRNAs involved in the modulation of myogenesis homeostasis in porcine skeletal muscle.

Results: In this study, a total of 655 novel individual IncRNAs including differentially expressed 24 IncRNAs, and 755 differentially expressed mRNAs were identified (fold change $\geq 2$ or $\leq 0.5$ and adjusted $P<0.05$ ). Integrated results of Gene Ontology (GO) and Kyoto Encyclopedia of Genes and Genomes (KEGG) enrichment analysis accompanied by the variation of intracellular $\mathrm{Ca}^{2+}$ concentration highlighted Lnc-ADAMTS9 involved in the modulation of myogenesis homeostasis in porcine skeletal muscle. Although Lnc-ADAMTS9 knock-down did not alter the mRNA expression of ADAMTS9, we demonstrated that Lnc-ADAMTS9 can promote myogenic proliferation and myogenic differentiation of myogenic precursors through inhibiting the ERK/MAPK signaling pathway.

Conclusion: We deciphered a comprehensive catalog of mRNAs and IncRNAs that might be involved in the regulation of myogenesis and adipogenesis homeostasis in the skeletal muscle of pigs. The Lnc-ADAMTS9 exerts an essential role in myogenesis through the ERK signaling pathway.
\end{abstract}

Keywords: Adipogenic precursors, LncRNA, Myogenic differentiation, Myogenic precursors, Myogenic proliferation, Pigs, Skeletal muscle

\footnotetext{
* Correspondence: yinjd@cau.edu.cn

State Key Laboratory of Animal Nutrition, College of Animal Science and Technology, China Agricultural University, No. 2 Yuanmingyuan West Road, Beijing 100193, China
}

C C The Author(s). 2021 Open Access This article is licensed under a Creative Commons Attribution 4.0 International License, which permits use, sharing, adaptation, distribution and reproduction in any medium or format, as long as you give appropriate credit to the original author(s) and the source, provide a link to the Creative Commons licence, and indicate if changes were made. The images or other third party material in this article are included in the article's Creative Commons licence, unless indicated otherwise in a credit line to the material. If material is not included in the article's Creative Commons licence and your intended use is not permitted by statutory regulation or exceeds the permitted use, you will need to obtain permission directly from the copyright holder. To view a copy of this licence, visit http://creativecommons.org/licenses/by/4.0/ The Creative Commons Public Domain Dedication waiver (http://creativecommons.org/publicdomain/zero/1.0/) applies to the data made available in this article, unless otherwise stated in a credit line to the data. 


\section{Introduction}

The skeletal muscle makes up $20 \%-50 \%$ of the total body mass in adult mammals and plays a pivotal role in regulating body metabolism and homeostasis [1]. The skeletal muscle not only implements the movement of bodies, but also serves as a tissue where ectopic lipid accumulated [2]. It is known that the adipocytes, myocytes, and fibroblasts all derive from mesenchymal progenitor cells during early embryonic development [3]. Myogenesis and adipogenesis occur competitively in the same microenvironment in skeletal muscle [4]. Furthermore, myogenesis is a crucial step for skeletal muscle development, regeneration, while excessive adipogenesis in skeletal muscle would suppress myogenesis and lead to myofibers infiltrated by lipids, and resultantly damages skeletal muscle contractive function and metabolism homeostasis [5]. In addition, the imbalance between myogenesis and adipogenesis in the skeletal muscle leads to various diseases including type-II diabetes, muscular atrophy, sarcopenia, and muscular dystrophy [6].

Myogenesis is regulated complicatedly by a cascade of intrinsic and extrinsic factors, including key transcriptional factors, miRNAs, and long non-coding RNAs (lncRNAs) [7], integrated by signaling pathways, such as Wnt/ $\beta$-catenin [8], Notch [9], and MAPK [10] pathways. However, mechanisms that mediate the balance between myogenic and adipogenic differentiation remain unclear.

Accumulating evidence have shown that lncRNAs are key regulators involved in numerous important biological processes including stem cell maintenance and differentiation, myogenesis, and adipogenesis in particular [11]. Recently, numbers of IncRNAs have been characterized in myogenesis. For instance, LncMyoD, activated by $M y o D$, plays an important role in promoting myogenesis and skeletal muscle regeneration [7]. Lnc$\mathrm{mg}$ is implicated in myogenesis and is required for MuSC differentiation by functioning as a competing endogenous RNA (ceRNA) of microRNA-125b in controlling IGF2 protein abundance [12]. In addition, Linc-RAM [13], MAR1 [14], and Neat1 [15] have been shown to play important roles in myogenesis, while a number of lncRNAs, such as MIR31HG [16] and TINCR [17], are implicated in adipogenesis. Therefore, the characterization of novel IncRNAs that mediate the balance between myogenesis and adipogenesis attracts intensive interest.

Sus scrofa provides a major source of animal-derived protein for humans as well as being an excellent model for understanding the balance between myogenesis and ectopic adipogenesis in the skeletal muscle, which determines muscle development potential and intramuscular fat accumulation in pigs [18]. Strengthening proliferation and differentiation potential of myogenic progenitor cells during fetal development promotes muscle growth and lean meat production while enhancing adipogenic commitment of adipogenic/fibrogenic progenitor cells in muscle improves marbling score and meat tenderness [19]. In this study, global transcriptional profiles of lncRNAs and mRNAs in myogenic and adipogenic precursor cells derived from porcine skeletal muscle were subjected to association analysis. Furthermore, a novel lncRNA XLOC_062039, located in the upstream of the ADAMTS9 gene, named Lnc-ADAMTS9, was identified as a critical lncRNA, which promoted myogenic proliferation and differentiation through the ERK signaling.

\section{Materials and methods \\ Cell isolation and culture}

The preplate technique established by our lab [20], was applied to isolate adipogenic and myogenic precursors from the longissimus dorsi muscle of neonatal Yorkshire pigs of 3-day-old from different litters $(n=3)$, purchased from Beijing Pig Breeding Center, Beijing, China. Briefly, after pigs being humanely killed, $1 \mathrm{~g}$ of muscle was sampled from the longissimus dorsi muscle, minced and digested in $0.17 \%$ protease (Sigma, P8811, MO, USA) and $0.15 \%$ collagenase-type XI (Sigma, C9407, MO, USA) solution for $1 \mathrm{~h}$, respectively. After filtered, centrifugated, and resuspended, the cell suspension was cultured in growth medium (GM) on a collagen I-coated dish at $37^{\circ} \mathrm{C}$ and $5 \% \mathrm{CO}_{2}$. GM containing DMEM/F12 (Hyclone, UT, USA) complemented with 10\% FBS (Gibco, 10099-141, CA, USA), $2 \mathrm{mmol} / \mathrm{L}$ glutamine (Gibco, CA, USA), antibiotics $(100 \mathrm{U} / \mathrm{mL}$ of penicillin and $100 \mathrm{mg} / \mathrm{mL}$ of streptomycin) and $5 \mathrm{ng} / \mathrm{mL}$ bFGF (basic fibroblast growth factor, PEPTECH, MA, USA). After $2 \mathrm{~h}$, non-adherent cells were transferred to another dish and further collected after $72 \mathrm{~h}$ adhering. The first $(0-2 \mathrm{~h})$ and second $(2-74 \mathrm{~h})$ sets of adherent cells were adipogenic and myogenic precursors, respectively. The medium was changed every 2 days.

\section{Cell transfection and treatments}

To explore the role of Lnc-ADAMTS9 in cell proliferation, myogenic precursors were plated on collagen Icoated six-well plates and transfected with $100 \mathrm{nmol} / \mathrm{L}$ scrambled siRNA or Lnc-ADAMTS9 siRNA (Ibsbio, Shanghai, China) using Lipofectamine 3000 (Invitrogen, Carlsbad, CA, USA) according to the manufacturer's protocol. After $48 \mathrm{~h}$ of transfection, cells were collected or induced for myogenic differentiation. Lnc-ADAMTS9 siRNA sequences were listed as follows: sense $5^{\prime}$-GCAA AUGUAUCAACGGGAUUU-3', antisense 5' -AUCCCG UUGAUACAUUUGCUU- $3^{\prime}$; The sequence of the scrambled siRNA was 5' - UUCUCCGAACGUGUCACGUTT-3' (sense strand) and 5'-ACGUGACACG UUCGGAGAATT-3' (antisense strand). 
U0126 (MedChem Express, HY-12031, Monmouth Junction, USA) was used as an ERK inhibitor [21] that depressed the phosphorylation of ERK1/2. To explore the role of Lnc-ADAMTS9 in myogenic differentiation, myogenic precursors were treated with scrambled siRNA + DMSO (Sigma, D2650, MO, USA), siLnc-ADAMTS9 + DMSO, and siLnc-ADAMTS9 + ERK inhibitor U0126 $(5 \mu \mathrm{mol} / \mathrm{L})$, respectively.

\section{Adipogenic and myogenic differentiation}

To evaluate the differential potentials of adipogenic and myogenic precursors, cells were subjected to adipogenic and myogenic induction, respectively. During adipogenic induction, precursor cells were treated with adipogenic differentiation medium (DM), including 10\% (v/v) FBS in DMEM, $1 \mu \mathrm{mol} / \mathrm{L}$ dexamethasone (Sigma, D4902, $\mathrm{MO}, \quad$ USA), $\quad 0.5 \mathrm{mmol} / \mathrm{L} \quad$ 1-methyl-3-isobutylmethylxanthine (Sigma, I5879, MO, USA), and $10 \mu \mathrm{g} / \mathrm{mL}$ insulin (Sigma, I6634, MO, USA). After $3 \mathrm{~d}$, the DM was replaced by the maintenance medium (DMEM complemented with $10 \% \mathrm{FBS}, 10 \mu \mathrm{g} / \mathrm{mL}$ insulin) for another $6 \mathrm{~d}$. As for myogenic induction, precursor cells at 80$90 \%$ confluence were switched to myogenic differentiation medium consisting of $2 \%$ heat-inactivated horse serum (Thermo Fisher, 16050130, DE, USA) in DMEM for $4 \mathrm{~d}$. Myogenic differentiation was assessed by the differentiation index and fusion index as described previously [22]. Differentiation index was calculated as the percentage of nuclei in myosin ${ }^{+}$cells. Fusion index was calculated as the percentage of nuclei contained in myotubes (myosin ${ }^{+}$cells with at least two nuclei).

\section{Oil red $O$ staining}

Lipid droplets were stained with Oil Red $\mathrm{O}$ to assess lipid accumulation and the efficiency of adipogenic differentiation. After the culture medium had been discarded, the cells were washed with $\mathrm{PBS}$ and $\mathrm{H}_{2} \mathrm{O}$ in turn. Subsequently, cells were fixed with $4 \%$ paraformaldehyde (PFA) for $30 \mathrm{~min}$ at room temperature (RT) and then incubated with $60 \%$ isopropanol for $5 \mathrm{~min}$, then removed from the isopropanol and shifted to the incubation with $2 \mathrm{~mL}$ of Oil Red O working solution / well for $30 \mathrm{~min}$ at room temperature. Finally, Oil Red O working solution was aspirated and the cells were washed with $\mathrm{H}_{2} \mathrm{O}$ before taking images. Oil Red O-stained lipids were eluted in $100 \%$ isopropanol, and the optical density (OD) was measured at $520 \mathrm{~nm}$.

\section{Immunofluorescence}

After myogenic induction, cells were fixed with 4\% PFA for $30 \mathrm{~min}$ at $\mathrm{RT}$, permeabilized with $0.2 \%$ Triton X-100 for $10 \mathrm{~min}$, blocked in blocking solution containing 5\% bovine serum albumin in PBS for $1.5 \mathrm{~h}$ and incubated with anti-MHC (MY32 clone, Sigma M4276, MO, USA) overnight at $4{ }^{\circ} \mathrm{C}$. After incubated with secondary antibody conjugated to Alexa Fluor 594. (ZSGB-BIO, ZF0513, Beijing, China) for $1 \mathrm{~h}$ at RT, cells were incubated with DAPI (Solarbio, C0065, Beijing, China) for $10 \mathrm{~min}$. Fluorescence was visualized using an Olympus fluorescence microscope (Olympus, Tokyo, Japan).

\section{Library preparation and sequencing analysis}

Total RNAs were extracted from cultured adipogenic precursors and myogenic precursors (from three neonatal pigs, respectively) using a HiPure Total RNA Mini Kit with DNA filter (Magen, Guangzhou, China). The quantity and quality of RNA were evaluated using a NanoDrop spectrophotometer (Thermo Scientific, Wilmington, DE, USA). Ribosomal RNA was removed from total RNA using the Ribo-zero rRNA Removal Kit (Illumina, San Diego, CA, USA). The sequencing libraries were constructed with the NEB Next ${ }^{\circ}$ Ultra $^{\mathrm{mm}}$ Directional RNA Library Prep Kit for Illumina (NEB, Ipswich, MA, USA) following the manufacturer's recommendations. Briefly, the obtained RNA was cut into short fragments and synthesized the first-strand cDNA using random hexamer primer. The second-strand cDNA was synthesized using DNA Polymerase I and RNase $\mathrm{H}$, in which dNTPs with dTTP were replaced by dUTP in the reaction buffer. After the adenylation of the 3' ends of DNA fragments, adaptor with hairpin loop structure was ligated to prepare for hybridisation. The library fragments were purified with AMPure XP system (Beckman Coulter, Beverly, MA, USA) for cDNA fragments selecting. The USER Enzyme (NEB, Ipswich, MA, USA) was used with size-selected, adaptor-ligated cDNA before PCR. Finally, products were purified and library quality was evaluated on the Agilent Bioanalyzer 2100 system (Agilent Technologies, Santa Clara, CA, USA). The libraries were sequenced on an Illumina HiseqX Ten platform (Illumina, San Diego, CA, USA) and 150-bp paired-end reads were generated.

\section{Raw data quality control and alignment}

The clean reads were obtained by filtering out the reads that contain adaptor or over $10 \%$ poly-Ns and over $50 \%$ of bases with Phred scores $<5$ from raw reads of fastq format. Moreover, Q20, Q30, and GC content of the clean reads were calculated. The reads that passed the quality control were mapped to the Sus scrofa genome (Sscrofa10.2) from Ensemble Sus using HISAT.

\section{Prediction of novel porcine IncRNAs}

High-quality reads were reconstructed to transcripts with StringTie (v1.0.4), and replicated transcripts were removed by Cuffcompare (v2.1.1). Subsequently, there are the following steps to predict the new IncRNA. Firstly, removing the transcripts with a total length of 
less than $200 \mathrm{nt}$. Secondly, filtering the 'background' transcripts for which the maximal expression was less than 2.0 and those present in only one sample or the number of exons was 1.0. Thirdly, the transcripts that overlapped with known mRNAs or lncRNAs on the same strand were discarded. Finally, the remaining transcripts without protein-coding potential jointly predicted by lncRNA prediction software Coding Potential Calculator (CPC, v0.9-r2), Pfam-scan (Pfam), and Coding Non Coding Index (CNCI, v2.1) were classified as lncRNAs.

\section{Differentially expressed mRNAs and IncRNAs}

The number of reads mapped to each gene was count by HRSeq v0.5.3. Reads per kilo bases per million reads (RPKM) were applied to quantify gene expression. The $\mathrm{R}$ package DESeq2 (v1.4.5) was employed to figure out the differentially expressed lncRNAs and mRNAs between adipogenic and myogenic precursors. The resulting $P$-values were adjusted for multiple testing using Benjamini and Hochberg's methods for controlling the false discovery rate (FDR). Fold change $\geq 2$ or $\leq 0.5$ and FDR $<0.05$ were set as the differentially expressed mRNAs or lncRNAs.

\section{Bioinformatics analysis}

The $\mathrm{R}$ package goseq (v1.16.2) was applied to perform the gene ontology (GO) enrichment analysis, and GO terms with a corrected $P \geq 0.05$ were excluded. For the Kyoto Encyclopedia of Genes and Genomes (KEGG) analysis, the differentially expressed genes (DEGs) were mapped directly to the KEGG database. Cutoff criteria were set with Benjamini-Hochberg false discovery rate $q<0.05$ for KEGG enrichment analysis.

LncRNAs exert regulative roles in the expression of mRNAs through direct competition with miRNA for binding loci or interaction with miRNA, known as mechanism of ceRNAs. In the present study, the putative interactions of lncRNA-miRNA and miRNA-mRNA were predicted by miRanda. Based on the predicted miRNA-mRNA and miRNA-lncRNA regulatory pairs, a ceRNA network was established in which the lncRNAs and mRNAs interacted via shared miRNAs. Cytoscape (Cytoscape Consortium, v.3.5.1, San Diego, CA, USA) were used to summarize and visualize ceRNA results. In order to investigate the function of IncRNAs, we predicted the cis target genes of differentially expressed lncRNAs by sequence homology (blastn, E-value $<1.0 \mathrm{E}$ 10 and identity $>99$ and matched length $\geq 20 \mathrm{bp}$ ) between gene and lncRNA pairs. Firstly, we chose coding genes locating in $10-100 \mathrm{~kb}$ upstream and downstream of IncRNAs. Subsequently, we calculated the Pearson correlation coefficient by Microsoft Excel (Redmond, WA, USA) between lncRNAs and corresponding genes. Finally, lncRNAs cis target genes were identified with the absolute value of the correlation coefficient more than 0.6.

\section{Quantitative real-time PCR analysis}

Total RNA was reverse-transcribed into cDNA using a PrimeScript RT reagent Kit with gDNA Eraser (Takara, RR047A, Japan) according to the manufacturer's instructions. SYBR Green based qPCR was performed in a qTOWER 2.2 thermocycler (Analytik Jena, Jena, Germany). All samples were measured in triplicate. The primers of selected genes were listed in Table S3. Glyceraldehyde 3-phosphate dehydrogenase (GAPDH) was selected as an internal control. Relative gene expression level was calculated by $2^{-\Delta \Delta \mathrm{Ct}}$ method [23].

\section{Intracellular calcium concentration measurement}

Intracellular $\mathrm{Ca}^{2+}$ concentration was measured by flow cytometry as described previously [24] with some modifications. In brief, cells were incubated in $5 \mu \mathrm{g} / \mathrm{mL}$ fluo-3 acetoxymethyl ester (Cayman Chemical, Ann Arbor, MI, USA) at $37^{\circ} \mathrm{C}$ for $30 \mathrm{~min}$ (protected from light) and then washed 3 times with PBS (containing 1\% FBS) and centrifuged at $1000 \mathrm{r} / \mathrm{min}$ for $5 \mathrm{~min}$. Subsequently, cells were resuspended in $200 \mu \mathrm{L}$ PBS (containing 1\% FBS). The conditions of the flow cytometer (BD Biosciences) were set to an excitation wavelength of $488 \mathrm{~nm}$ and a fluorescence signal acquisition wavelength of $525 \mathrm{~nm}$. After excluding debris and dead cells, 20,000 cells were collected in each sample collection gate and the average, and fluorescence intensity of the cells were calculated.

\section{Proliferation assay}

Myogenic precursors were plated into 96-well plates at a density of $5 \times 10^{3}$ cells. Cells at $70-80 \%$ confluence were incubated with 5-ethynyl-2'-deoxyuridine (EdU) for $2 \mathrm{~h}$ before staining. Cell proliferation was detected using Cell-Light EdU Cell Proliferation Detection Kit (Ribobio, C10310, Guangzhou, China) following the manufacturer's protocol. The percentage of proliferative cells was determined by quantitation of EdU-positive cells using an Olympus fluorescent microscope (Olympus, Tokyo, Japan).

\section{Flow cytometric analysis of cell cycle}

Cells cultured in six-well plates with $70-80 \%$ confluence were transfected for $48 \mathrm{~h}$, then fixed in $75 \%$ ethanol overnight at $-20^{\circ} \mathrm{C}$. Subsequently, the fixed cells were stained with propidium iodide $(50 \mu \mathrm{g} / \mathrm{mL})$ containing $10 \mu \mathrm{g} / \mathrm{mL}$ RNase A (Solarbio, Beijing, China), and then incubated for $30 \mathrm{~min}$ at $37^{\circ} \mathrm{C}$ in the dark. Flow cytometric analysis was performed with a flow cytometer (BD Biosciences) and data were processed by FlowJo 7.6 software. 


\section{Western blotting}

Total cell protein lysate was extracted with cell lysis buffer containing protease inhibitor and protein phosphatase inhibitor cocktail. The relative protein expression was analyzed by western blot. Approximately $60-100 \mu \mathrm{g}$ of total cell lysate was loaded and GAPDH was run as a loading control. In brief, protein samples were resolved on $8-12 \%$ SDS-PAGE gels and transferred to polyvinylidene fluoride membranes (Millipore, KGaA, Darmstadt, Germany). Membrane was blocked in TBS containing $5 \%(\mathrm{w} / \mathrm{v})$ skimmed milk powder or bovine serum albu$\min$ at RT for $1 \mathrm{~h}$ and then incubated against corresponding primary antibodies at $4{ }^{\circ} \mathrm{C}$ overnight include ERK (CST, 4695S, MA, USA), p-ERK (CST, 4370S, MA, USA) and GAPDH (CST, 5174S, MA, USA), respectively. Blots were developed using DyLight 800-labeled secondary antibodies, detected with the Odyssey $\mathrm{Clx}$ (4647 Superior Street, LI-COR Biotechnology, Lincoln, $\mathrm{NE}, \mathrm{USA})$ and quantified by ImageJ $(1.52 \mathrm{v}, \mathrm{NIH}, \mathrm{USA})$.

\section{Statistical analysis}

For comparison, data on lncRNA and mRNA between adipogenic and myogenic precursors were determined by the paired Student's $t$-test using SAS (version 9.2, $\mathrm{NC}$, USA), and the comparison concerning cell proliferation and differentiation were determined by Student's $t$ test as well. Data were presented as means \pm SEM. GraphPad Prism (version 8, CA, USA) was applied to graph. $P<0.05$ was considered significant.

\section{Results}

Characterization of adipogenic and myogenic precursors Upon adipogenic induction, adipogenic precursors differentiated into mature adipocytes, demonstrated by accumulating lipid droplets shown by Oil Red $\mathrm{O}$ staining (Fig. 1a, b). High mRNA expression levels of adipose- specific genes including peroxisome proliferatoractivated receptor $\gamma(P P A R \gamma, P=0.03)$, CCAAT-enhancer binding protein $\alpha(C E B P \alpha, P<0.01)$, and lipoprotein lipase $(L P L, P<0.01)$ were observed during the adipogenic differentiation of adipogenic precursors while little or no adipogenic potential was found in myogenic precursors (Fig. 1d).

Upon myogenic induction, myogenic precursors exhibited myogenic differentiation index (the proportion of myosin positive multi-nuclei myotubes), shown by immunofluorescence of myosin (Fig. 1a, c). A series of muscle-specific transcription factors, including myogenic factor 5 (MYF5, $P<0.01)$, myogenic differentiation 1 $(M Y O D 1, P<0.01)$, and myogenin (MYOG, $P=0.02)$, expressed more highly during the myogenic differentiation of myogenic precursors compared with those of adipogenic precursors (Fig. 1d). By contrast, adipogenic precursors exhibited very limited myogenic potential upon myogenic induction.

\section{Profile of mRNAs and IncRNAs in adipogenic and myogenic precursors}

Total RNA extracted from both adipogenic and myogenic precursors was subjected to high-throughput RNA-seq analysis following the workflow shown in Fig. 2a. We acquired 96 to 156 million and 89 to 111 million clean reads from the adipogenic and myogenic precursors, respectively (Table S1). In addition to 17,744 mRNAs identified, 775 putative long non-coding transcripts were identified (Fig. 2b), and among them, 27\%, $15 \%$, and $2 \%$ transcripts locate correspondingly in intergenic, intronic, and non-coding regions (Fig. 2c), representing 670 individual lncRNAs. After mapping to Sus scrofa genome from Ensembl, a total of 655 novel pig lncRNAs were discovered in the present study. The characteristics of IncRNA and mRNA concerning

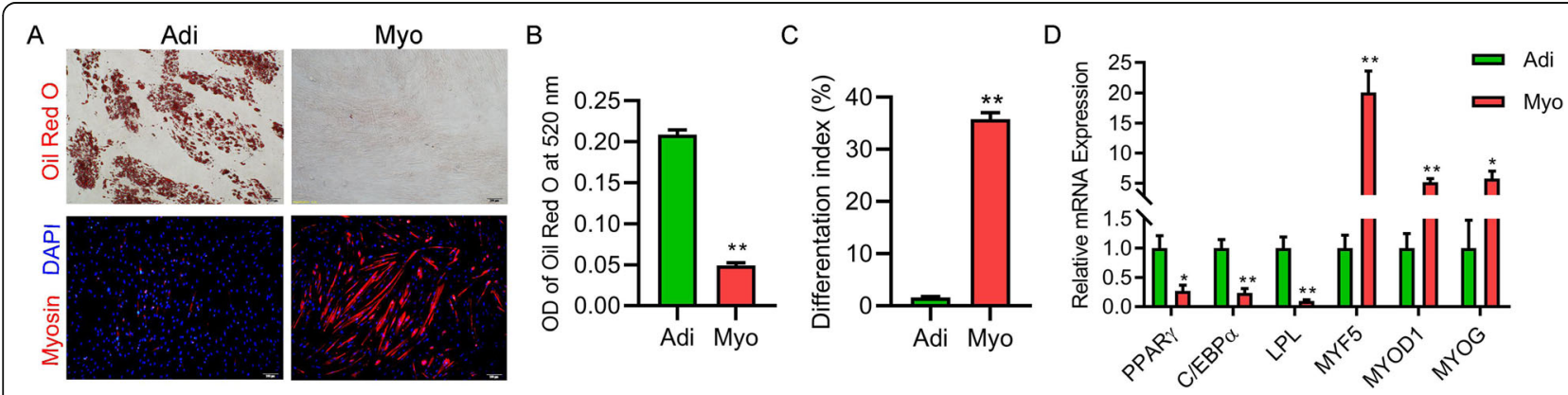

Fig. 1 Characterization of adipogenic (Adi-) and myogenic (Myo-) precursors derived from the skeletal muscle of neonatal pigs ( $n=3$ ). a Oil Red $\mathrm{O}$ staining and immunofluorescence of adipogenic and myogenic precursors following 9-d adipogenic induction and 3-d myogenic induction, respectively. Oil Red O (red), Myosin (red), and DAPI (blue). b OD of Oil Red O at $520 \mathrm{~nm}$ and differentiation index between Adi and Myo. c Differentiation index between Adi and Myo. d Quantitative RT-PCR for the mRNA expression level of adipogenesis specific genes (PPARY, C/EBPa, and $L P L$ ) and myogenesis specific genes (MYF5, MYOD1, and MYOG) following adipogenic or myogenic induction. Data are presented as mean \pm SEM. Student's $t$-test was used. Adi, adipogenic precursors; Myo, myogenic precursors. Scale bars, $100 \mu \mathrm{m} .{ }^{*} P<0.05,{ }^{*} P<0.01$ compared with adipogenic precursors 

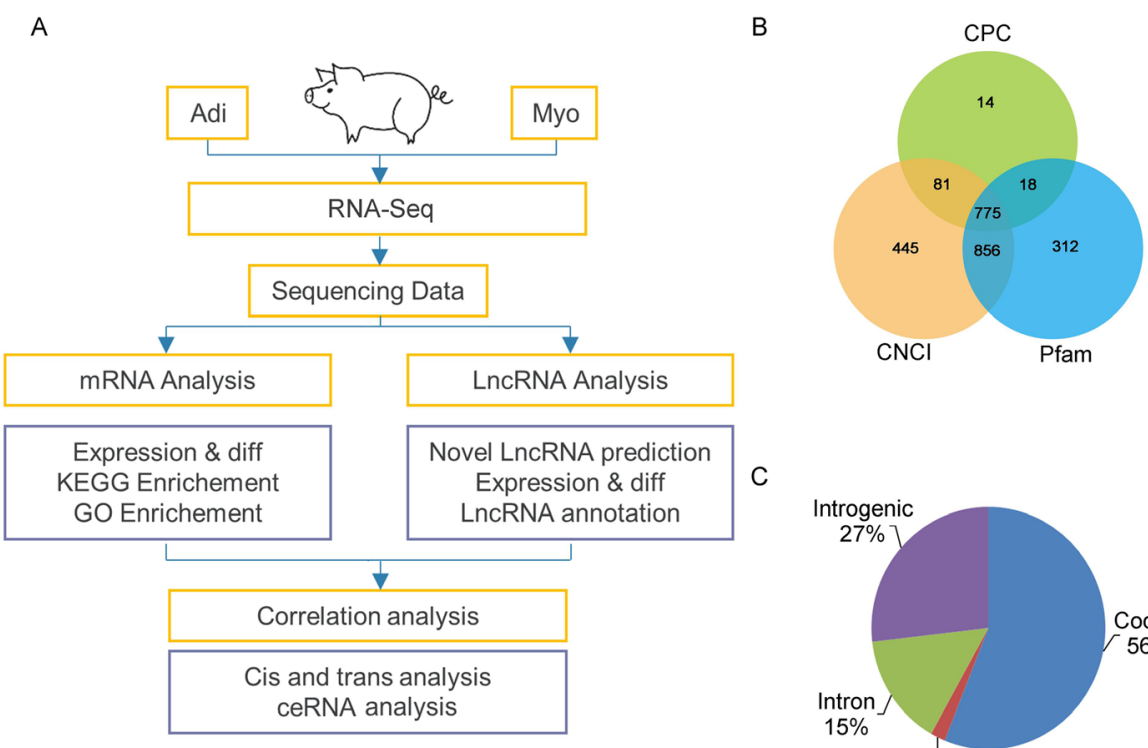

C

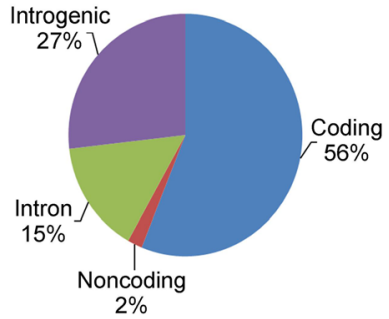

Fig. 2 Identification of IncRNAs in adipogenic (Adi-) and myogenic (Myo-) precursors ( $n=3$ ) a Workflow of high-throughput RNA-seq of adipogenic and myogenic precursors. b Non-coding transcripts identified by three kinds of software (CPC, CNCl, Pfam) were statistically analyzed and drew into a Venn diagram. c Pie charts representing the percentage of nucleotide bases mapping to indicated genomic regions

expression levels, exon numbers, open reading regions, sequence lengths, transcript numbers, and coding ability were shown in Fig. S1.

\section{Identification of differentially expressed mRNA and IncRNA}

The distributions of differentially expressed mRNAs and lncRNAs between myogenic and adipogenic precursors were shown in Fig. 3a. A total of 755 differentially expressed genes (DEGs) at mRNA level (Table S3) and 24 differentially expressed lncRNAs were identified between adipogenic and myogenic precursors. Among them, 572 DEGs were up-regulated while 183 DEGs were down-regulated in myogenic precursors compared with those in adipogenic precursors. Meanwhile, 9 lncRNAs were up-regulated and $15 \operatorname{lncRNAs}$ were down-regulated in myogenic precursors relative to adipogenic precursors (Fig. 3b; Table 1). Among these differentially expressed IncRNAs, XLOC_021529 and XLOC_001307 were the most up-regulated IncRNA while XLOC_155509 and XLOC_023175 were the most down-regulated lncRNA in myogenic precursors relative to adipogenic precursors. The heatmaps demonstrated the distinct expression pattern of the lncRNA and mRNA (Fig. 3c, d).

\section{Quantitative real-time PCR verification}

To validate the RNA-seq results, 9 differentially expressed lncRNAs (XLOC_012932, XLOC_189646,
XLOC_062039, XLOC_023175, XLOC_155509, XLOC_ 021811, XLOC_189950, XLOC_118028 and XLOC_ 119417) and 9 differentially expressed mRNAs (GLRB, PDGFC, TRIM55, PDGFD, MSTN, MEF2C, ADAMTS9, FGFR2 and ITGA9) were subjected to quantitative realtime PCR (Fig. 3e, f). The reliability of RNA-seq (transcriptome) analysis was validated by RT-qPCR (Pearson's $r=0.81)$ (Fig. 3g).

\section{GO enrichment analysis}

GO enrichment analysis showed that DEGs between adipogenic and myogenic precursors was annotated into 40 significant $\mathrm{GO}$ entries $(P<0.05)$, including 23 biological process (BP) GO entries, 13 cellular component (CC) GO entries, and 4 molecular function (MF) GO entries (Table S4). The top 30 significant GO terms (Fig. 4a) included calcium ion binding, extracellular space, the protein homodimerization activity, cytoskeleton, etc. Notably, GO entries related to muscle development, structure, and function, such as skeletal muscle tissue growth, structural constituent of muscle, and muscle contraction were significantly enriched $(P<0.05)$ (Fig. 4b). Among these terms, the majority of DEGs are up-regulated in myogenic precursors relative to adipogenic precursors.

\section{KEGG analysis}

A total of 37 pathways were significantly enriched based on DEGs between adipogenic and myogenic precursors 


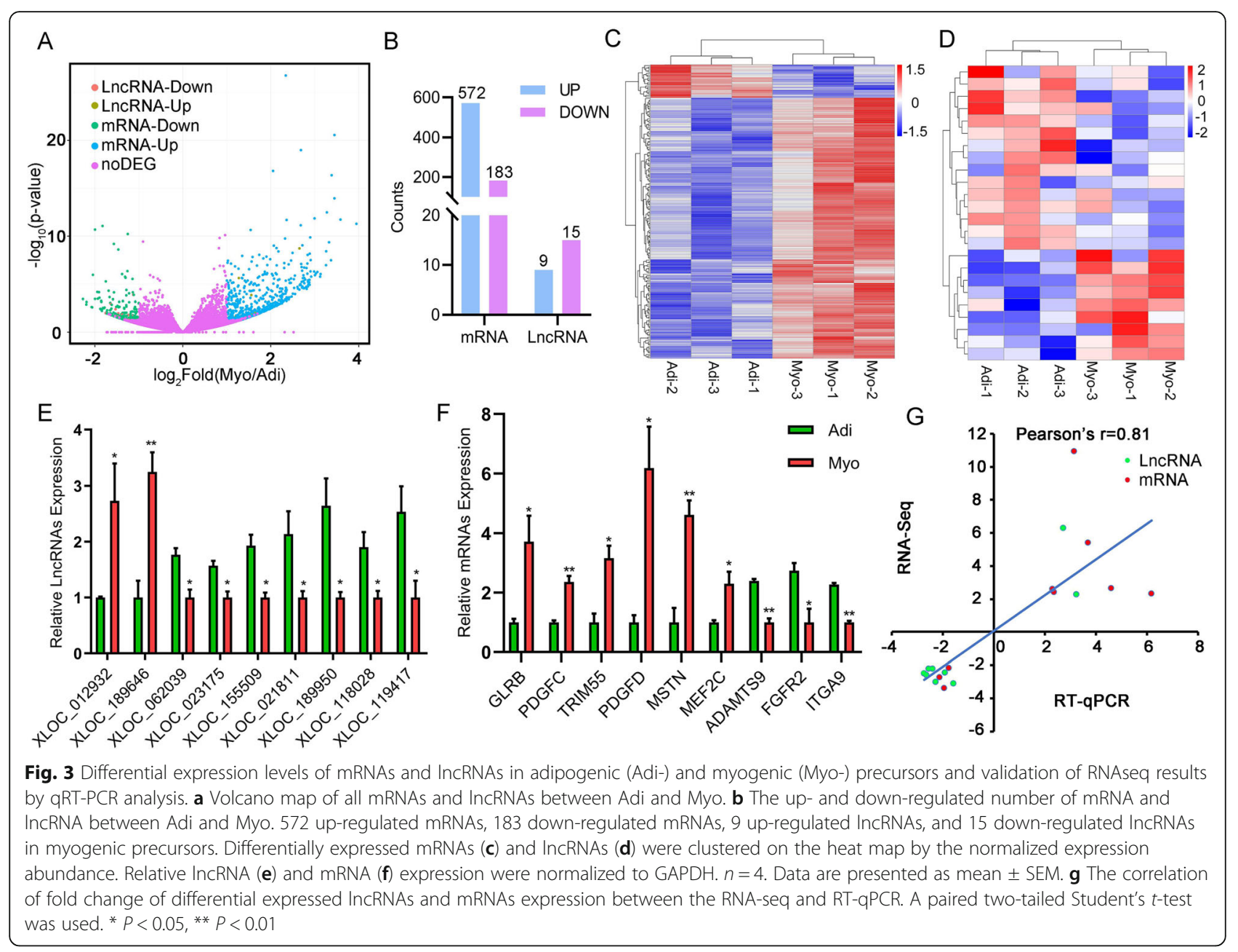

(Table S5). Among them, 20 significantly enriched pathways except those involved in human diseases were shown in Fig. 4c. In addition, Fig. S2 showed that DEGs, such as FGFR2, ITGA6, ITGA9, PDGFC, and PDGFD, acting as hubs to link important pathways including the Regulation of actin cytoskeleton, Focal adhesion, Calcium signaling pathway, MAPK signaling pathway, PI3K-Akt signaling pathway, cGMP-PKG signaling pathway, ECM-receptor interaction, Cytokine-cytokine receptor interaction and Signaling pathways regulating pluripotency of stem cells.

\section{Identification of candidate IncRNAs and putative ceRNA network}

The target genes of lncRNAs were predicted to explore the potential function of lncRNAs. The cis target genes of differentially expressed lncRNAs were shown in Table 2. There were 17 differentially expressed lncRNAs with adjacent genes. Among these adjacent genes, only ENSSSCG00000011496 (ADAMTS9), the adjacent gene of the lncRNA XLOC_062039, is a DEG between adipogenic and myogenic precursors. ADAMTS9 and lncRNA XLOC_062039, overlaps with a correlation coefficient of 0.98 , both of them are down-regulated in myogenic precursors relative to adipogenic precursors. We named XLOC_062039 as Lnc-ADAMTS9. Based on the common target miRNAs, we constructed a putative ceRNA mechanism series connecting lncRNAs and mRNAs network about the Lnc-ADAMTS9 (Fig. 4d). We discovered that Lnc-ADAMTS9 is an antisense transcript locating on $5^{\prime}$ of coding DNA sequence (CDS) of ADAMTS9 (Fig. 4e). A number of 6 miRNAs along with their linking 18 genes calculated in the present study should be explored in the future study.

\section{The key role of Lnc-ADAMTS9 in myogenic proliferation} and differentiation

To identify the role of Lnc-ADAMTS9 in myogenesis, we firstly investigated the role of Lnc-ADAMTS9 on myogenic proliferation. Lnc-ADAMTS9 knockdown in 
Table 1 Differentially expressed LncRNAs between in adipogenic and myogenic precursors

\begin{tabular}{|c|c|c|c|c|c|c|}
\hline Gene ID & Fold change & $P$-value & Chromosome & Strand & Exon number & Exon sizes, bp \\
\hline \multicolumn{7}{|l|}{ Up } \\
\hline XLOC_021529 & 6.29 & 1.92E-09 & Chr1 & + & 2 & $29 ; 202$ \\
\hline XLOC_012932 & 2.42 & 2.37E-06 & Chr9 & - & 1 & 1077 \\
\hline XLOC_079751 & 2.94 & $0.000,142$ & Chr14 & - & 2 & 190; 127 \\
\hline XLOC_090236 & 2.04 & $0.009,776$ & Chr15 & + & 2 & $17 ; 184$ \\
\hline XLOC_001307 & 3.13 & $0.010,441$ & Chr7 & + & 3 & $156 ; 104 ; 1671$ \\
\hline XLOC_164011 & 2.98 & $0.015,133$ & Chr6 & + & 1 & 259 \\
\hline XLOC_189646 & 2.28 & $0.025,817$ & Chr8 & + & 2 & $361 ; 114$ \\
\hline XLOC_091324 & 2.70 & $0.028,678$ & Chr15 & - & 1 & 304 \\
\hline XLOC_165070 & 2.68 & $0.035,623$ & Chr6 & - & 2 & $21 ; 131$ \\
\hline \multicolumn{7}{|l|}{ Down } \\
\hline XLOC_062039 & 0.46 & $0.000,132$ & Chr13 & + & 2 & $592 ; 149$ \\
\hline XLOC_023175 & 0.32 & $0.001,145$ & Chr1 & - & 2 & $229 ; 210$ \\
\hline XLOC_118825 & 0.35 & $0.003,995$ & Chr2 & - & 2 & $164 ; 431$ \\
\hline XLOC_155509 & 0.30 & $0.010,121$ & Chr5 & - & 2 & $1113 ; 182$ \\
\hline XLOC_021811 & 0.36 & $0.010,917$ & Chr1 & + & 2 & 1110; 783 \\
\hline XLOC_189950 & 0.38 & $0.014,067$ & Chr8 & + & 3 & $114 ; 114 ; 295$ \\
\hline XLOC_108084 & 0.42 & $0.016,205$ & Chr17 & - & 1 & 207 \\
\hline XLOC_189516 & 0.32 & $0.017,106$ & Chr8 & + & 2 & $52 ; 194$ \\
\hline XLOC_190244 & 0.35 & $0.019,752$ & Chr8 & - & 2 & 10,$531 ; 747$ \\
\hline XLOC_118028 & 0.41 & $0.019,867$ & Chr2 & + & 2 & 20,$801 ; 239$ \\
\hline XLOC_119417 & 0.45 & $0.020,062$ & Chr2 & - & 3 & $1785 ; 113 ; 121$ \\
\hline XLOC_154508 & 0.36 & $0.023,445$ & Chr5 & + & 2 & $427 ; 144$ \\
\hline XLOC_155480 & 0.36 & $0.025,117$ & Chr5 & - & 2 & $221 ; 42$ \\
\hline XLOC_178755 & 0.43 & $0.049,905$ & Chr7 & + & 2 & $662 ; 136$ \\
\hline XLOC_190092 & 0.41 & $0.049,938$ & Chr8 & - & 2 & 1858; 188 \\
\hline
\end{tabular}

myogenic precursors $(P<0.01$, Fig. 5a) did not alter the mRNA expression level of ADAMTS9, the cis target gene of Lnc-ADAMTS9 (Fig. 5b). Furthermore, siLncADAMTS9 decreased intracellular $\mathrm{Ca}^{2+}$ concentration compared with the scrambled ( $P=0.04$, Fig. $5 \mathrm{c})$. Meanwhile, the transfection of siLnc-ADAMTS9 significantly slowed cell proliferation $(P<0.01$, Fig. $5 d$, e). Flow cytometry analysis demonstrated that knockdown of LncADAMTS9 increased cell populations in the $G_{0} / G_{1}(P=$ $0.03)$ and $S$ phase $(P<0.01)$, and decreased cell population in the $G_{2}$ phase, resulting in arrested cells in the $G_{2}$ phase $(P<0.01$, Fig. 5f).

Besides, we also detected the role of Lnc-ADAMTS9 in myogenic differentiation. As a result, the knockdown of Lnc-ADAMTS9 remarkably decreased the mRNA expression of myoblast differentiation-specific genes including MYOD1 $(P=0.01)$, MYOG $(P<0.01)$, MYF5 $(P<0.01)$, and Myomaker $(P<0.01$, Fig. $5 \mathrm{~g})$ compared with the scrambled. Meanwhile, the knockdown of Lnc-
ADAMTS9 significantly blocked myotube formation (Fig. 5h), and depressed the differentiation index and fusion index compared with scrambled $(P<0.01$, Fig. 5i, j). Particularly, we observed that the knockdown of LncADAMTS9 significantly enhanced the phosphorylation of ERK $1 / 2$ during myogenic differentiation $(P<0.01$, Fig. $5 \mathrm{k}, 1)$. In order to investigate the role of the ERK signaling pathway in Lnc-ADAMTS9 mediated-myogenic differentiation, U0126, an ERK inhibitor, was employed to treat siLnc-ADAMTS9 cells. We observed that U0126 treatment significantly promoted myotube formation, and increased the differentiation index and fusion index compared with those of Lnc-ADAMTS9-knockdown cells $(P<0.01$, Fig. $5 \mathrm{~h}, \mathrm{i}, \mathrm{j})$. Meanwhile, the mRNA expression levels of genes involved in myoblast differentiation were also significantly increased upon U0126 treatment relative to that in siLnc-ADAMTS9 transfected myogenic precursors, whereas the ratio of $\mathrm{p}$-ERK/ ERK was significantly lowered $(P<0.01$, Fig. $5 k, 1)$. Thus, 


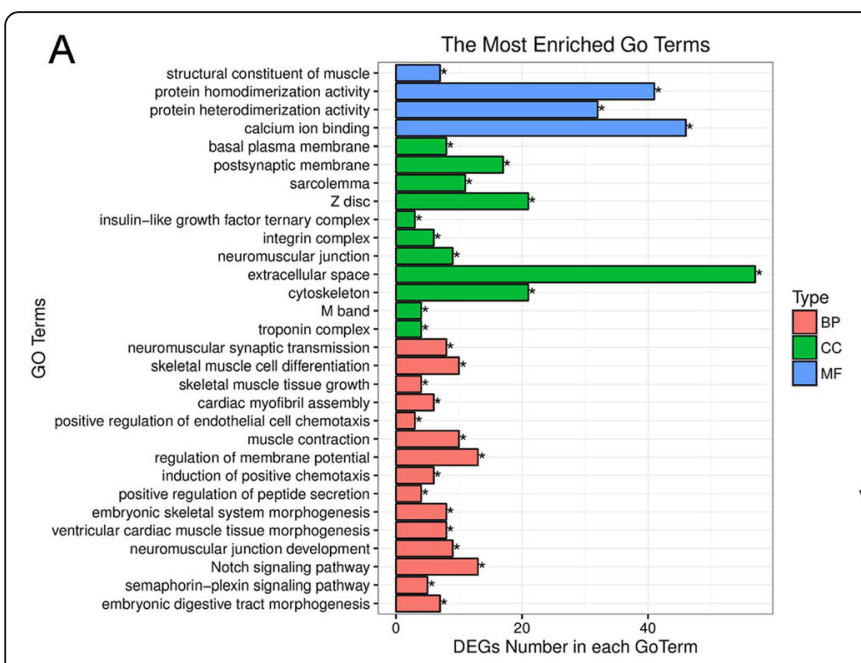

B

Significant muscle-related GO terms of DGEs

C
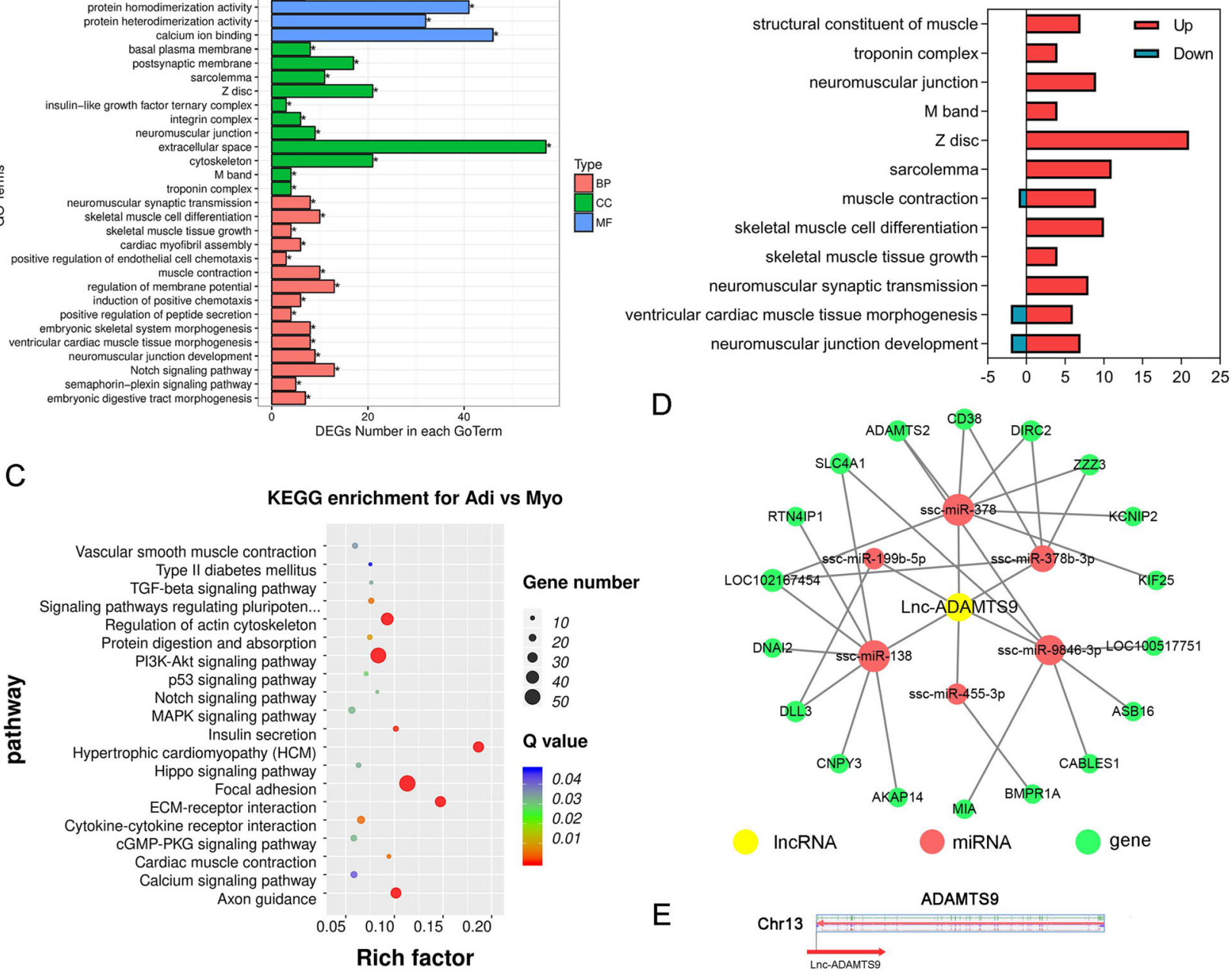

\section{D}

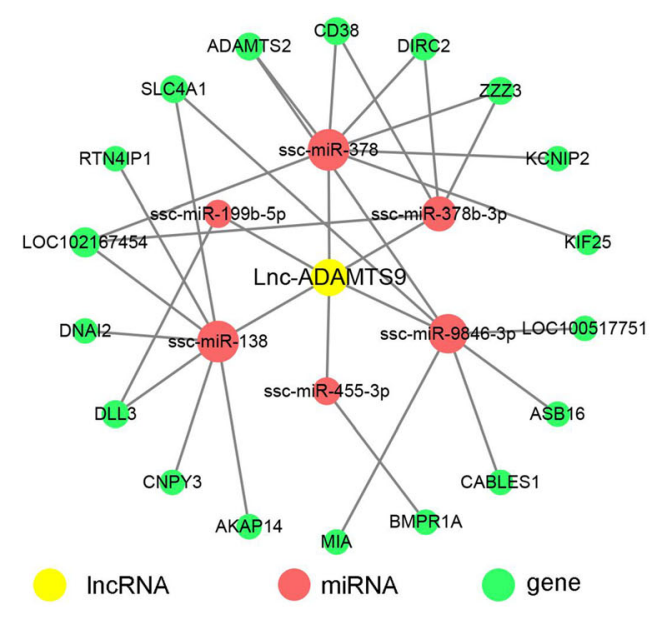

$\mathrm{E}$

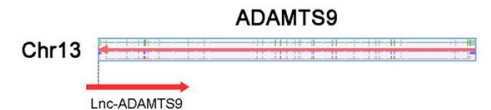

Fig. 4 Bioinformatics including GO and KEGG enrichment analysis of DEGs, ceRNA network, and the schematic diagram of Lnc-ADAMTS9. a The most enrichment of GO terms of DEGs. $\mathbf{b}$ The significant muscle-related GO terms of DEGs. $\mathbf{c}$ The bubble chart of KEGG pathway enrichment between DEGs. $\mathbf{d}$ ceRNA network of Lnc-ADAMTS9. e The schematic diagram of porcine ADAMTS9 and Lnc-ADAMTS9

Lnc-ADAMTS9 was required for myogenic proliferation and differentiation, and it can promote myogenic differentiation by inhibiting the ERK signaling pathway.

\section{Discussion}

Previous studies have demonstrated that lncRNAs take part in the modulation of skeletal muscle cell differentiation and muscle development in humans and mice [25]. For example, SYISL promotes myoblast proliferation and myotube fusion but inhibits myogenic differentiation through interacting directly with PRC2 [26]; IncRNA YY1 regulates skeletal muscle regeneration through modulating both mitochondrial functions and glycolytic pathways in satellite cells [27]. Although some lncRNAs were identified in pigs [28], databases concerning pig lncRNA information are very limited, and only a few lncRNAs whose functions had been revealed, for example, lnc_000414 was characterized closely related to adipogenesis and function as an inhibitor in the proliferation of intermyocellular preadipocytes [29]. In the present study, we investigated the roles of IncRNAs in the modulation of myogenesis and adipogenesis homeostasis in skeletal muscle by comparing global transcriptomic landmark between myogenic and adipogenic precursors derived from the skeletal muscle of pigs.

Firstly, we found that 755 DEGs between myogenic and adipogenic precursors, sharing 257 common DEGs with our previous study [20], and most DEGs were upregulated in myogenic precursors as well. Consistently, even more GO items were enriched in this study, the GO items concerning muscle constituents and functions, such as $\mathrm{Z}$ disc, $\mathrm{M}$ band, costamere, sarcolemma, basal plasma membrane, structural constituent of muscle, skeletal muscle contraction, and muscle filament sliding 
Table 2 Cis target genes of differentially expressed IncRNAs

\begin{tabular}{lllllll}
\hline LncRNAs & LncRNA fold change & mRNA & Gene symbol & mRNA fold change & Relationship, bp & Correlation index \\
\hline XLOC_021529 & 6.29 & ENSSSCG00000005087 & SIX 1 & 1.94 & Downstream:23,747 & 0.96 \\
XLOC_079751 & 2.94 & ENSSSCG00000009880 & SLC8B1 & 1.12 & Overlap & 0.61 \\
XLOC_164011 & 2.98 & ENSSSCG00000003102 & PNMA8A & 0.60 & Upstream:22,659 & -0.91 \\
XLOC_189646 & 2.28 & ENSSSCG00000021506 & - & 3.21 & Downstream:72,987 & 0.88 \\
XLOC_165070 & 2.68 & ENSSSCG00000026699 & ZNF180 & 1.06 & Downstream:41,893 & 0.72 \\
XLOC_062039 & 0.46 & ENSSSCG00000011496 & ADAMTS9 & 0.45 & Overlap & 0.98 \\
XLOC_023175 & 0.32 & ENSSSCG00000005751 & COL5A1 & 0.75 & Overlap & 0.64 \\
XLOC_155509 & 0.30 & ENSSSCG00000000837 & CHST11 & 0.98 & Overlap & 0.78 \\
XLOC_021811 & 0.36 & ENSSSCG00000005491 & ATP6V1G1 & 1.08 & Downstream:17,273 & -0.88 \\
& & ENSSSCG00000005492 & TMEM268 & 0.88 & Overlap & 0.76 \\
XLOC_189950 & 0.38 & ENSSSCG00000009230 & WDFY3 & 0.71 & Overlap & 0.91 \\
XLOC_108084 & 0.42 & ENSSSCG00000007391 & MATN4 & 1.67 & Upstream:6245 & -0.70 \\
XLOC_189516 & 0.32 & ENSSSCG00000008725 & CYTL1 & 0.88 & Downstream:15,131 & 0.90 \\
XLOC_118028 & 0.41 & ENSSSCG00000029992 & NAV2 & 0.55 & Overlap & 0.98 \\
XLOC_119417 & 0.45 & ENSSSCG00000024043 & ADAMTS2 & 0.80 & Intron & 0.83 \\
XLOC_154508 & 0.36 & ENSSSCG00000000191 & KMT2D & 0.56 & Downstream:27,338 & 0.65 \\
XLOC_155480 & 0.36 & ENSSSCG00000030685 & ARID2 & 0.77 & Intron & 0.64 \\
XLOC_178755 & 0.43 & ENSSSCG00000002526 & RCOR1 & 0.67 & Overlap & 0.99 \\
\hline
\end{tabular}

were similar to our previous study. Regarding cell differentiation and fate determination, GO items including skeletal muscle cell differentiation and embryonic skeletal system morphogenesis were also annotated by DEGs in this study. Accordingly, some similar GO items concerning cell components of extracellular matrix and cell adhesion, such as ECM-receptor interaction, cytoskeleton, calcium ion binding, and protein homodimerization/heterodimerization activity were also enriched. Notably, we presently annotated GO items including neuromuscular junction development and neuromuscular synaptic transmission, implying that neuromuscular junction exerts a critical role in the balance between myogenesis and adipogenesis in skeletal muscle, which warrants continual study in the future.

Aside from enriched pathways shown by our previous study, in the present study, KEGG analysis showed some pathways were implicated with both adipogenesis and myogenesis, such as focal adhesion, ECM-receptor interaction, PI3K-Akt signaling pathway, regulation of actin cytoskeleton, MAPK signaling pathway, and calcium signaling pathway. Studies have shown that focal adhesion protein levels are inversely related to embryonic stem cell adipogenic capacity, and increased levels of focal adhesion proteins inhibit lipogenesis in embryonic stem cells [30]. Cytoskeleton mechanics plays an important role in the balance of myogenic and adipogenic differentiation [31]. Hub genes involved in these pathways might be implicated in the distinct potential of adipogenic- and myogenic precursors. For example, FGFR4 can attenuate satellite cell differentiation during postnatal development [32] and its deficiency may improve glucose metabolism and insulin sensitivity in mice [33].

$\mathrm{Ca}^{2+}$ is necessary for cell proliferation and $\mathrm{G}_{1}$ is the most sensitive phase of the cell cycle to $\mathrm{Ca}^{2+}$ depletion [34]. Intracellular $\mathrm{Ca}^{2+}$ concentrations and calcium signaling pathways are important factors in regulating cell proliferation and differentiation [35, 36]. Intracellular $\mathrm{Ca}^{2+}$ activates the MAPK pathway and resultantly participates in stem cell differentiation into lineages $[37,38]$. Consistently, we had evidence that intracellular $\mathrm{Ca}^{2+}$ and the calcium signaling pathway, as well as the MAPK signaling pathway, are advantageous for Myo-lineage cells to keep a potent differentiation potential [20, 24, 39].

As well known, the mammalian family of MAPK includes ERK, p38, and JNK. It had been indicated that ERK dimerizes in response to phosphorylation [40]. Preventing the dimerization of ERK diminishes the ERK cytoplasmic signaling [41]. ERK dimers are mainly detected in the cytoplasm and are related to scaffold proteins that serve as platforms of ERK dimerization, through which ERK binds to cytoplasmic substrates [42]. In addition to significantly enriched GO term protein homodimerization/heterodimerization activity, we also found that seven key DEGs between myogenic precursors and adipogenic precursors, including PDE6C, CCL21, FGFR2, FGFR4, ALOX15, ADCYAP1, and FGF10, positively regulate ERK1 and ERK2 cascades, 


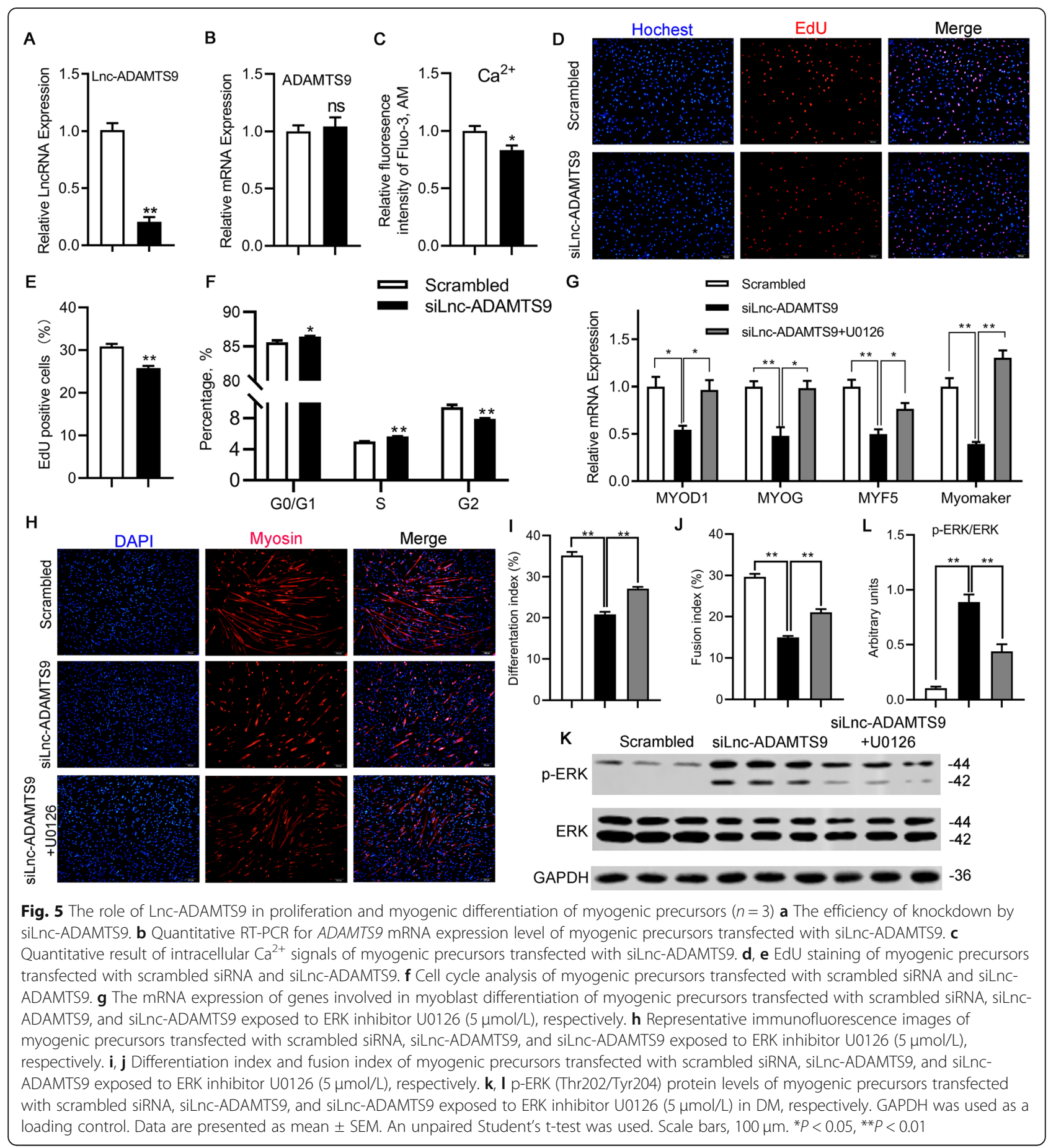

implying that the ERK/MAPK pathway may be a powerful candidate mechanism underlying the modulation of myogenesis and adipogenesis homeostasis in porcine skeletal muscle.

It had been supposed that lncRNA probably expressed parallel to the adjacent mRNAs on the same chain [43]. Previous studies had suggested that lncRNAs modulated the expression of adjacent genes in a trans- and/or cis- acting way [44]. In the present study, we identified that two IncRNAs putatively targeted ADAMTS9 and SIX1, respectively, implicated in the determination of the distinct differential potential of adipogenic- and myogenic precursors. SIX1 has been considered as the main determinant of fast-twitch fiber type acquisition and maintenance [45] and binds to adipogenic and brown adipose tissue marker genes during adipogenesis [46]. 
Furthermore, lncRNA-Six1 can regulate SIX1 expression in a ceRNA-dependent way [47] or encodes a micropeptide to activate the SIX1 gene [48].

ADAMTS9, locating in cell surface/pericellular matrix proteolysis in diverse contexts, is a highly conserved secreted protease that remodels provisional ECM in morphogenetic processes [49] and takes part in the regulation of skeletal muscle insulin sensitivity [50]. In humans, ADAMTS9-AS2 was identified as an antisense transcript of the ADAMTS9 [51]. ADAMTS9-AS2 inhibits cell proliferation via the miR-27a-3p/FOXO1 axis in clear cell renal cell carcinoma [52] and controls the chondrogenic differentiation in human mesenchymal stem cells through a ceRNA mechanism [53]. In the present study, we originally speculated that Lnc-ADAM TS9 may function through ADAMTS9 in a lncRNA/ mRNA pair manner. However, ADAMTS9 mRNA expression was not altered by the knocked down of LncADAMTS9.

To investigate the role of Lnc-ADAMTS9 in proliferation and differentiation of myogenic precursors, we detected the effect of knockdown of Lnc-ADAMTS9 on proliferation and differentiation of myogenic precursors. We found that Lnc-ADAMTS9 knockdown decreased intracellular $\mathrm{Ca}^{2+}$ concentration. $\mathrm{Ca}^{2+}$ serves as a second messenger. Increased intracellular $\mathrm{Ca}^{2+}$ had been shown to play a critical role not only in myogenic differentiation but also in a variety of cellular and physiological functions like cell cycle progression and apoptosis [54]. Therefore, we deduced that Lnc-ADAMTS9 may upregulate intracellular $\mathrm{Ca}^{2+}$ and promote myogenesis through cooperating with $\mathrm{Ca}^{2+}$, which warrants further validation. Meanwhile, in the present study, we observed that LncRNA-ADAMTS9 knockdown reduced the proliferation of myogenic precursors accompanied by the increased cell population in the $G_{1}$ and $S$ phase and decreased cell population in the $G_{2}$ phase, indicating that Lnc-ADAMTS9 plays a key role in promoting myogenic precursor proliferation.

Besides, ERK1/2 cascade plays an opposite role in proliferation and myogenic differentiation by suppressing the expression of muscle-specific genes [55]. The controversy exists on the role of ERK in myogenic differentiation. For example, FGF13 activated the ERK1/2 pathway to down-regulate Spry1 protein expression and lead to $\mathrm{C} 2 \mathrm{C} 12$ cell differentiation inhibition [56]. However, EPHA2 was required for myogenic differentiation, and it may promote myogenic differentiation through ERK signaling [57]. In the present study, we observed that Lnc-ADAMTS9 knockdown activated the ERK1/2 pathway, while depressed myogenic differentiation and impaired myotube formation. Moreover, ERK inhibitor U0126 can recover the impaired myogenic differentiation potential of myogenic precursors induced by
siLnc-ADAMTS9 knockdown. It strongly supported that Lnc-ADAMTS9 promoted myogenic differentiation through depressing the ERK1/2 pathway, and ERK1/2 negatively alter myogenic differentiation of myogenic precursors derived from the skeletal muscle of pigs.

Above all, it is conceivable that Lnc-ADAMTS9 serves as a positive role in promoting proliferation and myogenic differentiation of myogenic precursors through the regulation of the ERK1/2 pathway.

\section{Conclusions}

We provided a comprehensive catalog of mRNAs and lncRNAs involved in the regulation of myogenesis and adipogenesis homeostasis in the skeletal muscle of pigs. A novel functional lncRNA, named Lnc-ADAMTS9, was identified and characterized to be implicated in the modulation of proliferation and myogenic differentiation of myogenic precursors. We proved that Lnc-ADAM TS9 is required for proliferation, myogenic differentiation of myogenic precursors through mediating the ERK signaling pathway. The role of Lnc-ADAMTS9 in adipogenesis warrants future study, which embodies the significance of intramuscular fat accumulation in pigs.

\section{Supplementary Information}

The online version contains supplementary material available at https://doi. org/10.1186/s40104-020-00524-4.

Additional file 1: Table S1. Summary of reads mapping to the reference genome.

Additional file 2: Table S2. Primer sequences used in qRT-PCR analysis. Additional file 3: Figure S1. Comparison of features of IncRNAs and mRNAs between myo- and adio-precursors. A) The comparison of IncRNA and mRNA expression levels. B) The comparison of IncRNA and mRNA exon number. C) The comparison between IncRNA and mRNA open reading region. D) Comparison of the length of IncRNA and mRNA transcripts. E) The comparison of the number of IncRNA and mRNA transcripts. F) The comparison of the coding ability scores of known IncRNA, mRNA, and new IncRNA.

Additional file 4: Figure S2. Network of hub DEGs in key KEGG pathways. Ovals represent hub DGEs and diamonds represent KEGG pathways. Networks were visualized by Cytoscape (v3.5.1).

Additional file 5: Table S3. List of 755 DEGs between adipogenic and myogenic precursors derived from pig skeletal muscle. Table S4. List of GO terms based on 755 DEGs between adipogenic and myogenic percursors derived from pig skeletal muscle. Table S5. List of KEGG terms based on 755 DEGs between adipogenic and myogenic percursors derived from pig skeletal muscle.

Additional file 6: Graphical abstract.

\section{Abbreviations}

Adi: Adipogenic precursors; CDS: Coding DNA sequence; ceRNA: Competing endogenous RNA; DEG: Differentially expressed gene; DM: Differentiation medium; EdU: 5-ethynyl-2'-deoxyuridine; FDR: False discovery rate; GM: Growth medium; GO: Gene Ontology; KEGG: Kyoto Encyclopedia of Genes and Genomes; LncRNA: Long non-coding RNA; Myo: Myogenic precursors; OD: Optical density; PFA: Paraformaldehyde; RPKM: Reads per kilo bases per million reads; RT: Room temperature 


\section{Acknowledgements}

Not applicable.

\section{Authors' contributions}

JDY conceived, designed the experiments, and obtained the finance supports; LQW, TH, KQ, XZ and YBW mainly performed the experiments; LQW, YBW and NJ analyzed the data; LQW and TH wrote the manuscript; $L Q W, L J H$ and JDY revised the manuscript. All authors read and approved the final manuscript.

\section{Funding}

This study was financially supported by the National key research and development program of China (Grant No. 2018YFD0500402), the National Natural Science Foundation of China (Grant No. 31790412, Grant No. 31672431), and the National Key Basic Research Program of China (2013CB127302)

\section{Availability of data and materials}

All data generated or analyzed during this study are included in this published article [and its supplementary information files].

\section{Ethics approval and consent to participate}

All procedures conducted in the present study were approved by the Institutional Animal Care and Use Committee of China Agriculture University (ID: SKLAB-B-2010-003)

\section{Consent for publication}

Not applicable.

\section{Competing interests}

The authors declare that they have no competing interests.

Received: 21 June 2020 Accepted: 16 November 2020

Published online: 02 February 2021

\section{References}

1. Li Y, Chen $X$, Sun $H$, Wang $H$. Long non-coding RNAs in the regulation of skeletal myogenesis and muscle diseases. Cancer Lett. 2018;417:58-64.

2. Uezumi A, Ikemoto-Uezumi M, Tsuchida K. Roles of nonmyogenic mesenchymal progenitors in pathogenesis and regeneration of skeletal muscle. Front Physiol. 2014,5:68.

3. Du M, Wang B, Fu X, Yang Q, Zhu M. Fetal programming in meat production. Meat Sci. 2015;109:40-7.

4. Yin H, Price F, Rudnicki MA. Satellite cells and the muscle stem cell niche. Physiol Rev. 2013;93:23-67.

5. Takegahara Y, Yamanouchi K, Nakamura K, Nakano S, Nishihara M. Myotube formation is affected by adipogenic lineage cells in a cell-to-cell contactindependent manner. Exp Cell Res. 2014;324:105-14.

6. Alfonso JCJ, Avan AS. Sarcopenia. Lancet. 2019;393:2636-46.

7. Gong C, Li Z, Ramanujan K, Clay I, Zhang Y, Lemire BS, et al. A long noncoding RNA, LncMyoD, regulates skeletal muscle differentiation by blocking IMP2-mediated mRNA translation. Dev Cell. 2015:34:181-91.

8. Sah JP, Hao NTT, Han X, Tran TT, McCarthy S, Oh Y, et al. Ectonucleotide pyrophosphatase 2 (ENPP2) plays a crucial role in myogenic differentiation through the regulation by WNT/ $\beta$-catenin signaling. Int J Biochem Cell Biol. 2020;118:105661

9. Brack AS, Conboy IM, Conboy MJ, Shen J, Rando TA. A temporal switch from notch to Wnt signaling in muscle stem cells is necessary for normal adult myogenesis. Cell Stem Cell. 2008;2:50-9.

10. Yuan $Y, X u Y, X u$ J, Liang B, Cai $X$, Zhu C, et al. Succinate promotes skeletal muscle protein synthesis via Erk1/2 signaling pathway. Mol Med Rep. 2017; 16:7361-6.

11. Chen R, Lei S, Jiang T, Zeng J, Zhou S, She Y. Roles of IncRNAs and circRNAs in regulating skeletal muscle development. Acta Physiol (Oxford). 2020;228: e13356

12. Zhu M, Liu J, Xiao J, Yang L, Cai M, Shen H, et al. Lnc-mg is a long noncoding RNA that promotes myogenesis. Nat Commun. 2017:8:14178.

13. Yu X, Zhang Y, Li T, Ma Z, Jia H, Chen Q, et al. Long non-coding RNA LincRAM enhances myogenic differentiation by interacting with MyoD. Nat Commun. 2017:8:14016.
14. Zhang ZK, Li J, Guan D, Liang C, Zhuo Z, Liu J, et al. A newly identified IncRNA MAR1 acts as a miR-487b sponge to promote skeletal muscle differentiation and regeneration. J Cachexia Sarcopenia Muscle. 2018;9:61326.

15. Wang S, Zuo H, Jin J, Lv W, Xu Z, Fan Y, et al. Long noncoding RNA Neat1 modulates myogenesis by recruiting Ezh2. Cell Death Dis. 2019;10:505.

16. Huang Y, Jin C, Zheng Y, Li X, Zhang S, Zhang Y, et al. Knockdown of IncRNA MIR31HG inhibits adipocyte differentiation of human adiposederived stem cells via histone modification of FABP4. Sci Rep-UK. 2017;7: 8080.

17. Liu Y, Wang Y, He X, Zhang S, Wang K, Wu H, et al. LncRNA TINCR/miR-31$5 \mathrm{p} / \mathrm{C} / \mathrm{EBP}$-a feedback loop modulates the adipogenic differentiation process in human adipose tissue-derived mesenchymal stem cells. Stem Cell Res. 2018;32:35-42.

18. Bassols A, Costa C, Eckersall PD, Osada J, Sabrià J, Tibau J. The pig as an animal model for human pathologies: a proteomics perspective. Proteomics Clin Appl. 2014;8:715-31.

19. Du M, Huang Y, Das AK, Yang Q, Duarte MS, Dodson MV, et al. Meat Science and Muscle Biology Symposium: manipulating mesenchymal progenitor cell differentiation to optimize performance and carcass value of beef cattle. J Anim Sci. 2013;91:1419.

20. Sun W, He T, Qin C, Qiu K, Zhang X, Luo Y, et al. A potential regulatory network underlying distinct fate commitment of myogenic and adipogenic cells in skeletal muscle. Sci Rep UK. 2017;7:44133.

21. Ohno Y, Oyama A, Kaneko H, Egawa T, Yokoyama S, Sugiura T, et al. Lactate increases myotube diameter via activation of MEK/ERK pathway in C2C12 cells. Acta Physiol. 2018;223:e13042.

22. Millay DP, O'Rourke JR, Sutherland LB, Bezprozvannaya S, Shelton JM, Olson EN. Myomaker is a membrane activator of myoblast fusion and muscle formation. Nature. 2013;499:301-5.

23. Schmittgen TD, Livak KJ. Analyzing real-time PCR data by the comparative C(T) method. Nat. Protoc. 2008;3(6):1101-8.

24. Qiu K, Xu D, Wang L, Zhang X, Jiao N, Gong L, Yin J. Association analysis of single-cell RNA sequencing and proteomics reveals a vital role of $\mathrm{Ca}^{2+}$ signaling in the determination of skeletal muscle development potential. Cells. 2020;9:1045.

25. Legnini I, Morlando M, Mangiavacchi A, Fatica A, Bozzoni I. A feedforward regulatory loop between HuR and the long noncoding RNA linc-MD1 controls early phases of Myogenesis. Mol Cell. 2014;53:506-14.

26. Jin JJ, Lv W, Xia P, Xu ZY, Zheng AD, Wang XJ, et al. Long noncoding RNAS YISL regulates myogenesis by interacting with polycomb repressive complex 2. P Natl Acad Sci USA. 2018;115:e9802-11.

27. Chen F, Zhou J, Li Y, Zhao Y, Yuan J, Cao Y, et al. YY1 regulates skeletal muscle regeneration through controlling metabolic reprogramming of satellite cells. EMBO J. 2019:38:e99727.

28. Zou C, Li L, Cheng X, Li C, Fu Y, Fang C, et al. Identification and functional analysis of long Intergenic non-coding RNAs underlying intramuscular fat content in pigs. Front Genet. 2018;9:102.

29. Sun Y, Chen X, Qin J, Liu S, Zhao R, Yu T, et al. Comparative analysis of long noncoding RNAs expressed during intramuscular adipocytes Adipogenesis in fat-type and lean-type pigs. J Agric Food Chem. 2018;66:12122-30.

30. Szabo E, Feng T, Dziak E, Opas M. Cell adhesion and spreading affect Adipogenesis from embryonic stem cells: the role of Calreticulin. Stem Cells. 2009;27:2092-102.

31. McBeath R, Pirone DM, Nelson CM, Bhadriraju K, Chen CS. Cell shape, cytoskeletal tension, and RhoA regulate stem cell lineage commitment. Dev Cell. 2004;6:483-95.

32. Zhang $W, X u Y$, Zhang $L$, Wang $S$, Yin B, Zhao S, et al. Synergistic effects of TGFB2, WNT9a, and FGFR4 signals attenuate satellite cell differentiation during skeletal muscle development. Aging Cell. 2018;17:e12788.

33. Ge H, Zhang J, Gong Y, Gupte J, Ye J, Weiszmann J, et al. Fibroblast growth factor receptor 4 (FGFR4) deficiency improves insulin resistance and glucose metabolism under diet-induced obesity conditions. J Biol Chem. 2014;289: 30470-80.

34. Kahl CR, Means AR. Regulation of cell cycle progression by calcium / Calmodulin-dependent pathways. Endocr Rev. 2003;24:719-36.

35. Schaar A, Sun Y, Sukumaran P, Rosenberger TA, Krout D, Roemmich JN, et al. $\mathrm{Ca}^{2+}$ entry via TRPC1 is essential for cellular differentiation and modulates secretion via the SNARE complex. J Cell Sci. 2019;132:231878.

36. Filip S, Mokrý J, Forostyak O, Dayanithi G. The extracellular matrix and $\mathrm{Ca}(2+)$ signaling mechanisms. Physiol Res. 2019;68:161-70. 
37. Almalki SG, Agrawal DK. ERK signaling is required for VEGF-A / VEGFR2induced differentiation of porcine adipose-derived mesenchymal stem cells into endothelial cells. Stem Cell Res Ther. 2017;8:113.

38. Gao J, Fan M, Xiang G, Wang J, Zhang X, Guo W, et al. Diptoindonesin G promotes ERK-mediated nuclear translocation of p-STAT1 (Ser727) and cell differentiation in AML cells. Cell Death Dis. 2017;8:-e2765.

39. Qiu K, Zhang X, Wang L, Jiao N, Xu D, Yin J. Protein expression landscape defines the differentiation potential specificity of Adipogenic and myogenic precursors in the skeletal muscle. J Proteome Res. 2018;17:3853-65.

40. Herrero A, Pinto A, Colón-Bolea P, Casar B, Jones M, Agudo-lbáñez L, et al. Small molecule inhibition of ERK dimerization prevents tumorigenesis by RAS-ERK pathway oncogenes. Cancer Cell. 2015;28:170-82.

41. Casar B, Pinto A, Crespo P. Essential role of ERK dimers in the activation of cytoplasmic but not nuclear substrates by ERK-scaffold complexes. Mol Cell. 2008:31:708-21.

42. Santos E, Crespo P. The RAS-ERK pathway: A route for couples. Sci Signal. 2018;11:eaav0917.

43. Cabili MN, Trapnell C, Goff L, Koziol M, Tazon-Vega B, Regev A, et al. Integrative annotation of human large intergenic noncoding RNAs reveals global properties and specific subclasses. Genes Dev. 2011;25:1915-27.

44. Hung T, Chang HY. Long noncoding RNA in genome regulation. RNA Biol. 2010:5:582-5.

45. Sakakibara I, Wurmser M, Dos Santos M, Santolini M, Ducommun S, Davaze $\mathrm{R}$, et al. Six 1 homeoprotein drives myofiber type IIA specialization in soleus muscle. Skelet Muscle. 2016;6:30.

46. Brunmeir R, Wu J, Peng X, Kim S, Julien SG, Zhang Q, et al. Comparative Transcriptomic and Epigenomic analyses reveal new regulators of murine Brown Adipogenesis. PLoS Genet. 2016;12:e1006474.

47. Ma M, Cai B, Jiang L, Abdalla BA, Li Z, Nie Q, et al. IncRNA-Six 1 is a Target of miR-1611 that Functions as a ceRNA to Regulate Six1 Protein Expression and Fiber Type Switching in Chicken Myogenesis. Cells. 2018;7:243.

48. Cai B, Li Z, Ma M, Wang Z, Han P, Abdalla BA, et al. LncRNA-Six1 encodes a micropeptide to activate Six 1 in Cis and is involved in cell proliferation and muscle growth. Front Physiol. 2017;8:230.

49. Enomoto H, Nelson CM, Somerville RPT, Mielke K, Dixon LJ, Powell K, et al. Cooperation of two ADAMTS metalloproteases in closure of the mouse palate identifies a requirement for versican proteolysis in regulating palatal mesenchyme proliferation. Development. 2010;137:4029-38.

50. Graae A, Grarup N, Ribel-Madsen R, Lystbæek SH, Boesgaard T, Staiger H, et al. ADAMTS9 regulates skeletal muscle insulin sensitivity through extracellular matrix alterations. Diabetes. 2019;68:502-14.

51. Yao J, Zhou B, Zhang J, Geng P, Liu K, Zhu Y, et al. A new tumor suppressor LncRNA ADAMTS9-AS2 is regulated by DNMT1 and inhibits migration of glioma cells. Tumor Biol. 2014;35:7935-44.

52. Song EL, Xing L, Wang L, Song W, Li D, Wang Y, et al. LncRNA ADAM TS9-AS2 inhibits cell proliferation and decreases chemoresistance in clear cell renal cell carcinoma via the miR-27a-3p/FOXO1 axis. Aging. 2019:11:5705-25.

53. Huang MJ, Zhao JY, Xu JJ, Li J, Zhuang YF, Zhang XL. IncRNA ADAMTS9-AS2 controls human mesenchymal stem cell chondrogenic differentiation and functions AS a ceRNA. Mol Ther Nucleic Acids. 2019;18:533-45.

54. Cook SJ, Lockyer PJ. Recent advances in $\mathrm{Ca}^{2+}$-dependent Ras regulation and cell proliferation. Cell Calcium. 2006;39:101-12.

55. Bennett AM, Tonks NK. Regulation of distinct stages of skeletal muscle differentiation by mitogen-activated protein kinases. Science. 1997;278: 1288-91.

56. Lu H, Shi X, Wu G, Zhu J, Song C, Zhang Q, et al. FGF13 regulates proliferation and differentiation of skeletal muscle by down-regulatingSpry1. Cell Prolif. 2015:48:550-60.

57. Zhang X, Wang L, Qiu K, Xu D, Yin J. Dynamic membrane proteome of adipogenic and myogenic precursors in skeletal muscle highlights EPHA2 may promote myogenic differentiation through ERK signaling. FASEB J. 2019:33:5495-509.

Ready to submit your research? Choose BMC and benefit from:
- fast, convenient online submission
- thorough peer review by experienced researchers in your field
- rapid publication on acceptance
- support for research data, including large and complex data types
- gold Open Access which fosters wider collaboration and increased citations
- maximum visibility for your research: over 100M website views per year
At BMC, research is always in progress.
Learn more biomedcentral.com/submissions

